\section{An APL program for measures of association for ordinal variables}

\author{
JERRY M. BRENNAN, LARRY H. NITZ, \\ and JENNIFER E. DALY \\ University of Hawaii, Honolulu, Hawaii
}

A variety of measures of association can be used to establish the degree of relationship between two ordinal variables. In many of these methods, researchers consider pairs of observations on two ordinal variables and determine whether knowledge of the rank order on one variable is useful in predicting the rank order on the second variable. In these predictions, knowledge of the nature of the association between the ordinal pairs can reduce errors. Specifically, if the association between the pairs is positive, the same rank order would be predicted on the second variable as the pair had on the first variable. Conversely, if the association is negative, the opposite rank order would be predicted on the second variable as the pair had on the first variable. These two rules are referred to as the same rank order and opposite rank order, respectively (Loether \& McTavish, 1976, p. 221).

To implement these rules, researchers must establish which rule, either same or opposite rank order, would yield the most accurate prediction. This is accomplished by computing the total number of unique pairs possible and establishing the patterns of pairings present in the data. Five pairings are possible: concordant pairs $\left(N_{\mathfrak{A}}\right)$, pairs that are ranked on the same order on both variables; discordant pairs $\left(N_{d}\right)$, pairs that are ranked on the opposite order on both variables; pairs tied on the independent variable $(X)$ but not on the dependent variable $(Y)$; pairs tied on the dependent variable $(Y)$ but not on the independent variable $(X)$; and pairs tied on both variables. Ordinal measures of association indicate simple ratios, with the preponderance of like- versus opposite-ranked pairs $\left(N_{s}-N_{d}\right)$ indicating the reduction in error that can be achieved by using one of the rank-order prediction rules (i.e., same or opposite rank order) (Loether \& McTavish, 1976, p. 222).

Although the numerator of these ordinal measures remains the same (i.e., the number of like- versus oppositeranked pairs), the denominator of the ratio varies according to the types of pairs being predicted. Six formulas are used to adjust the numerator pursuant to the type of association being predicted: Kendall's (1955) tau-a $\left(t_{\mathrm{a}}\right)$, tau-b $\left(t_{b}\right)$, and tau-c $\left(t_{c}\right)$; Goodman and Kruskal's $(1963,1972)$ gamma (G); and Somers's (1962) $d_{x y}$ and $d_{y x}$ (see Appendix A).

Address correspondence to Jerry M. Brennan, Department of Sociology, University of Hawaii, Honolulu, HI 96822.
Tau-a indicates the prevalence of concordant or discordant pairs relative to all other pairings in the data. This formula yields a symmetrical coefficient, since neither the numerator nor the denominator takes into account any ties in the paired data. Tau-b establishes an average of the two Somers's $d s$. This formula is also symmetrical and produces a coefficient that indicates the strength of the association among pairings. Tau-b is used more frequently than tau-a because it takes into account nontrivial pairings; however, due to the complexity of the denominator, operational definitions based on this measure are difficult to state (Loether \& McTavish, 1976, p. 230). In addition, tau-b cannot achieve 1.0 when the number of rows and columns in the table are unequal (i.e., rectangular tables). Therefore, in such cases, another measure of association, tau-c, can be used to adjust the numerator and the denominator of the ratio according to the smaller number of rows or the smaller number of columns in a table. Unfortunately, as with tau-b, the tau-c formula, although normed, is also troublesome to interpret. For this reason, this measure is used less frequently than the other tau measures (Loether \& McTavish, p. 230).

Goodman and Kruskal's $(1963,1972)$ gamma uses the same numerator as does tau-a, but the denominator removes any ties found in the pairings, producing a coefficient that reflects only untied pairings. Somers's (1962) $d_{x y}$ and $d_{y x}$ are used when ties are being predicted between the independent and dependent variable; the formula employed depends upon the direction of the prediction. Utilization of Somers's $d_{x y}$ versus Somers's $d_{y x}$ depends upon the variable chosen as the independent variable. Generally, however, the two Somers's $d s$ can be calculated for any table, and both formulas produce asymmetrical coefficients that predict the ranking of the pairs on the independent or dependent variable based on knowledge of existing ties on one of the two variables.

ORCOEF is a noniterative program that computes and displays all of the above-mentioned coefficients (Appendix B). The main program, ORCOEF, calls ORPAIR, which determines the preponderance of like- versus opposite-ranked pairs and the number of unique pairings present in the data. ORPAIR calls SUB, a utility function, which sums the subsets of the data matrices. Finally, ORCOEF computes and displays the coefficients.

Program Input. The input to ORCOEF is a crosstabulation table of frequencies. There are no limits to the dimensions of the two-way table.

Program Output. The output consists of (1) the number of concordant and discordant pairs present in the data $\left(N\right.$, and $\left.N_{d}\right)$; (2) the number of pairs tied on the independent, dependent, and both variables $\left(T_{x}, T_{y}\right.$, and $T_{x y}$, respectively); (3) the total number of unique pairings in the data $(T)$; and (4) the six ordinal coefficients $\left(t_{a}, t_{b}\right.$, $t_{c}, G, d_{x y}$, and $\left.d_{y x}\right)$. 
Language. The programs are written in STSC APL, Versions 5.1 and above, for the IBM PC; however, the program should run with most versions of APL.

Additional Comments. The example shown in Appendix B is identical to the one found in Loether and McTavish $(1976$, p. 223 ); however, each variable is ordered from low to high.

Availability. A program listing of ORCOEF is available without charge. Copies of the program on diskette may be obtained by sending a blank diskette to the first author.

\section{REFERENCES}

GoOdman, L. A., \& KRUSKaL, W. H. (1963). Measures of association for cross classifications: III. Approximate sampling theory. Journal of the American Statistical Association, 58, 310-364.

Goodman, L. A., \& KrUSKAL, W. H. (1972). Measures of association for cross classification: IV. Simplification of asymptotic variances. Joumal of the American Statistical Association, 67, 415-421.

KENDALL, M. G. (1955). Rank correlation methods. New York: Hafner.

LOETHER, H. J., MCTAVISH, D. G. (1976). Descriptive and inferential statistics: An introduction (pp. 221-232). Boston, MA: Allyn \& Bacon.
SOMERs, R. H. (1962). A new asymmetric measure of association for ordinal variables. American Sociological Review, 27, 799-811.

\section{APPENDIX A}

Formulas Used by ORCOEF to Compute Ordinal Coefficients

$$
\begin{aligned}
\text { Tau-a }\left(t_{a}\right) & =\frac{N_{d}-N_{d}}{T} \\
\text { Tau-b }\left(t_{b}\right) & =\sqrt{d_{x y} d_{y z}} \\
\text { Tau-c }\left(t_{c}\right) & =\frac{2 m\left(N_{t}-N_{d}\right)}{N^{2}(m-1)} \\
\text { Gamma }(G) & =\frac{N_{d}-N_{d}}{N_{d}+N_{d}} \\
\text { Somers's } d_{x y} & =\frac{N_{d}-N_{d}}{N_{d}+N_{d}+T_{x}} \\
\text { Somers's } d_{y x} & =\frac{N_{d}-N_{d}}{N_{d}+N_{d}+T_{y}}
\end{aligned}
$$

\section{APPENDIX B}

Functions Used in the Calculation of Ordinal Coefficients

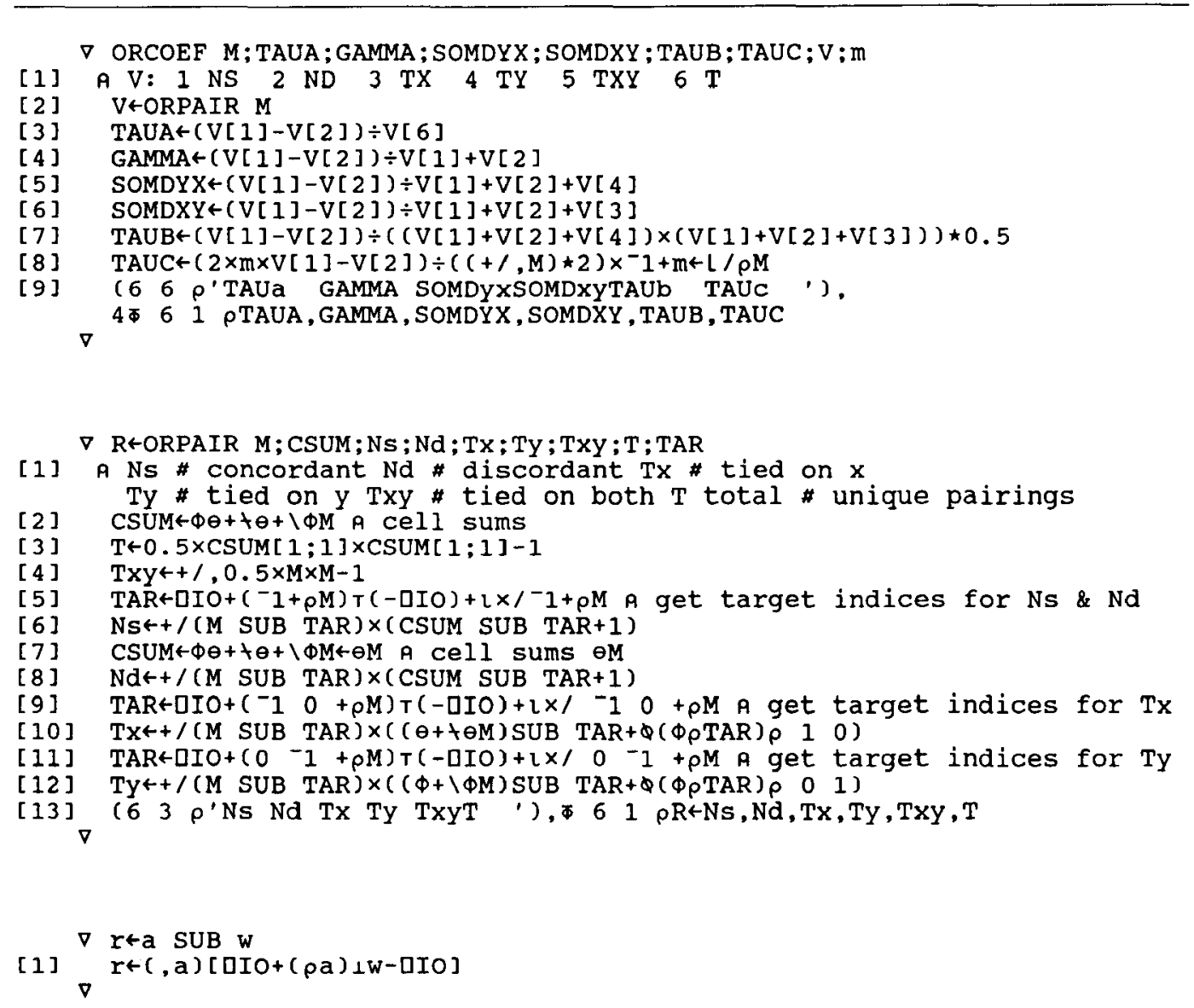


EXAMPLE :

Enter 3 by 3 matrix of frequencies as a vector.

Reshape to 3 by 3 and call function ORCOEF.

$\begin{array}{lllllllllllll}\text { ORCOEF } & 3 & 3 & \rho & 71 & 37 & 30 & 59 & 53 & 48 & 29 & 55 & 68\end{array}$

and the results are:
Ns $\quad 31057$
Nd $\quad 14705$
TX 21614
Ty 21677
TXY 11972
T 101025
TAUa $\quad .1619$
GAMMA .3573
SOMDYX $\quad .2425$
SOMDXY $\quad .2427$
TAUb $\quad .2426$
TAUC $\quad .2423$

Note-ORCOEF is the main program. ORPAIR is a function that determines the preponderance of like-ranked versus oppositeranked pairs and the number of unique pairings present in the data. SUB is a utility function that sums the subsets of the data matrices.

(Revision accepted for publication March 18, 1988.) 